\title{
Non-passivated manganese-based carbonaceous nanoparticles selectively target kidney outer medulla, exhibiting renal clearance, high T1- weighted MRI contrast, subtle citotoxicity and low opsonization level.
}

\section{Yarima Garcia}

Centro de estudos avanzados de Cuba

\section{Thais Menezes}

Universidade Federal de Pernambuco

Marcela Barros

Universidade Federal de Pernambuco

Elizabeth da Silva

Universidade Federal de Pernambuco

\section{Lucas Maggi}

Universidade Federal de Pernambuco

\section{Gustavo Ventura}

Universidade Federal de Pernambuco

Rafaela de Queiroz

Universidade Federal do Rio de Janeiro

\section{Andréia Santos}

Universidade Federal do Rio de Janeiro

\section{Patricia Cruz}

Universidade Federal do Rio de Janeiro

Wagner Dias

Universidade Federal do Rio de Janeiro

\section{Susana Frases}

Universidade Federal do Rio de Janeiro

Adriane Todeschini

Universidade Federal do Rio de Janeiro Jorge Neves ( $\square$ jorge.Ineves@ufpe.br) Universidade Federal de Pernambuco 
Keywords: contrast, agent, MRI, CQD, manganese, in vivo

Posted Date: March 12th, 2021

DOI: https://doi.org/10.21203/rs.3.rs-318737/v1

License: (c) (1) This work is licensed under a Creative Commons Attribution 4.0 International License. Read Full License 


\title{
Non-passivated manganese-based carbonaceous nanoparticles selectively target kidney outer medulla, exhibiting renal clearance, high T1-weighted MRI contrast, subtle citotoxicity and low opsonization level.
}

\author{
Yarima Sanchez Garcia $^{1,4}$, Thais Meira Menezes ${ }^{1}$, Marcela Rodrigues Barros ${ }^{1}$, Elizabeth Martins da Silva ${ }^{1}$, Lucas \\ Oliveira Maggi ${ }^{2}$, Gustavo Tavares Ventura ${ }^{3}$, Rafaela Muniz de Queiroz ${ }^{3}$, Andreia Vasconcelos dos Santos ${ }^{3}$, Patricia \\ Carvalho Cruz $^{3}$, Wagner Barbosa Dias ${ }^{3}$, Susana Frases ${ }^{3}$, Adriane Regina Todeschini ${ }^{3}$, and Jorge Luiz Neves ${ }^{* 1}$ \\ ${ }^{1}$ Departamento de Química Fundamental, CCEN, Universidade Federal de Pernambuco, Recife 50670-901, PE, Brazil \\ ${ }^{2}$ Centro de Informática, CIn, Universidade Federal de Pernambuco, Recife 50670-901, PE, Brazil \\ ${ }^{3}$ Instituto de Biofísica, CCS, Universidade Federal do Rio de Janeiro, Rio de Janeiro 21944-970, RJ, Brazil \\ ${ }^{4}$ Centro de estudos avanzados de Cuba, CEA, Valle Grande, La Lisa 17100, La Habana, Cuba ${ }^{\dagger}$
}

\begin{abstract}
Nanoparticle passivation by molecular recognition moieties (small molecules, aptamers, antibodies) imputes unique characteristics to nanoparticles (NPs) and unlocks their fascinating selectivity to targeting disease sites. Although non-passivated nanomaterials may archive selective targeting by exploring NP features and their interaction with biofluids proteins, such studies are scarce in the literature. Here, we report spherical manganese-based carbonaceous NPs (MnCQD), which specifically target mice kidneys after intravenous injection, even without direct surface chemical modification. Also, the NPs provide high image contrast in $\mathrm{T}_{1}$ magnetic resonance imaging (MRI) with subtle toxicological effects. The unexpected selectivity of MnCQD to the kidney has been examined based on their determined intrinsic properties and their interaction with two blood proteins: Bovine Serum Albumin (BSA), and Human Transferrin (HTF). More particularly, aspects such as size, composition, superficial charge, spectroscopic features, interaction mechanism, affinities, thermodynamic, and protein structural changes have been investigated. All these results highlight the excellent potential of MnCQD as a low toxic $\mathrm{T}_{1^{-}}$ MRI contrast agent and open the prospect of using non-functionalized NPs as a selective agent to target specific organs.
\end{abstract}

Keywords: contrast, agent, MRI, CQD, manganese, in vivo.

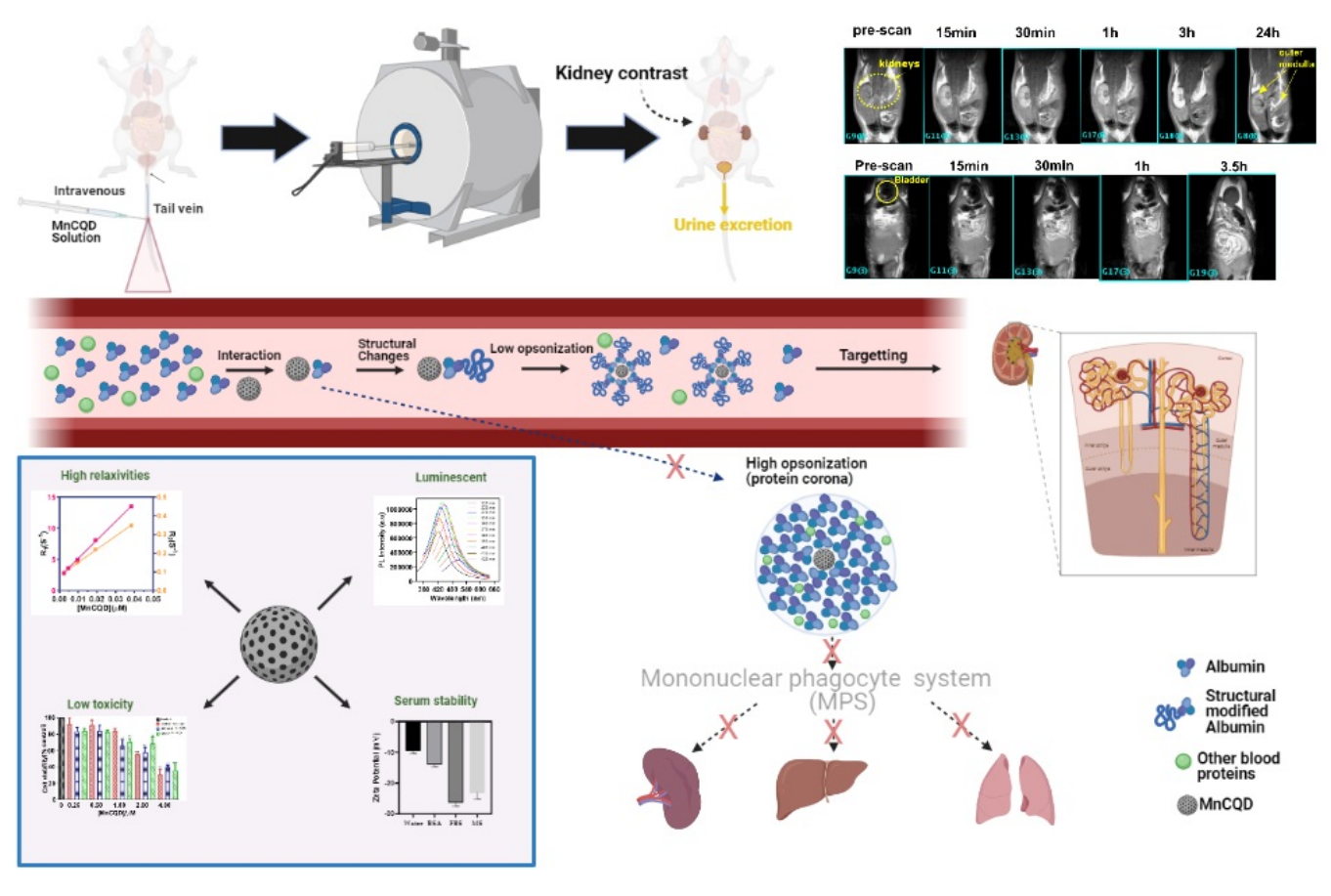

\footnotetext{
${ }^{*}$ Corresponding author: jorge.Ineves@ufpe.br

†Email: ysanchezg10@gmail.com (Yarima Sanchez Garcia), thais_meira22@hotmail.com (Thais Meira Menezes), Marcela.Jacinta@hotmail.com (Marcela Rodrigues Barros), martinselizabeth685@gmail.com (Elizabeth Martins da Silva), lucasomaggi@gmail.com (Lucas Oliveira Maggi), gustavotventura@hotmail.com (Gustavo Tavares Ventura), munizrq@gmail.com (Rafaela Muniz de Queiroz), andreia@biof.ufrj.br (Andréia Vasconcelos Santos), p.carvcruz@gmail.com (Patricia Carvalho Cruz), wdias1976@gmail.com (Wagner Barbosa Dias), susanafrases@biof.ufrj.br (Susana Frases), adritodeschini@biof.ufrj.br (Adriane Regina Todeschini), jorge.lneves@ufpe.br (Jorge Luiz Neves)
} 
Metal encapsulation confers additional NP features and allows nanocomposite to be applied in diverse areas $^{1}$. However, these untargeted metal-containing NPs can undergo fast clearance by the reticuloendothelial system, which leads the NPs to organ accumulation for lengthened periods and also grows concerns about their biodistribution and toxicity ${ }^{2}$. Although NP properties can be designed to extend blood circulation, nonspecific biodistribution is still inevitable ${ }^{3}$. Although renal excretion could be an alternative due to their size-dependent attributes, coating the NPs to reach the renal system can also block the NP renal excretion ${ }^{4}$. It is indubitable the vital role of various physicochemical properties to determine the biological fate of the NPs. In addition to the size, NP surface opsonization seems to be a determinant and critical parameter for NP localization ${ }^{5}$. Opsonization is a natural process and leads the NP surface primarily to be covered with proteins (opsonins) presented in biological fluids and occurs when the NP surface is in contact with fluids like blood. A protein corona around the NP surface is then constituted by the proteic opsonins, which labels the exogenous NPs to be destroyed by the mononuclear phagocyte system (MPS) before they can target the selected tissue or organ and perform the designed task. In this regard, the accumulation of such untargeted NPs frequently occurs in MPS organs, more prominently the liver and spleen. Rare studies were intending to selective targeting organs like kidneys. Aspiring to provide surface protection, NPs are typically covered with unique non-opsonizing materials like polyethylene glycol (PEG), a process called PEGylation, which delivers stealth NPs capable of avoiding the MPS and reaching the desired organ ${ }^{6}$. Besides non-opsonization materials, a recent study demonstrates that bovine serum albumin (BSA) structural modification (due NP-protein interaction) leads the protein to inhibit opsonized cationic polystyrene NPs from attaching to cellular receptors of phagocytic cells ${ }^{7}$. In contrast, the cationic NP can enhance their cellular attachment.

Magnetic resonance imaging (MRI) contrast agents are crucial tools to enhance disease diagnosis and treatment ${ }^{8}$. The most vital properties of MRI contrast agents are their intrinsic paramagnetism ${ }^{9}$, and their high relaxation rates ${ }^{10}$. Nevertheless, for diagnostic purposes, these compounds must also have low toxicity as well as a rapid and complete excretion after medical examination ${ }^{11}$. Currently, the most commonly utilized contrast agents are gadolinium and manganese complexes ${ }^{12}$. Meanwhile, gadolinium-based MRI imaging probes are linked to the onset of nephrogenic systemic fibrosis (NSF), rare but severe, and potentially fatal fibrosis of the skin and internal organs that can arise in renal impaired patients ${ }^{13}$. More recently, several reports have identified dose-dependent gadolinium accumulation in the brains of patients with a normal renal function that has received contrast-enhanced MRI ${ }^{14}$, raising further concerns by the FDA regarding this class of compounds. Gadolinium retention in a subset of patients further underscores the need for gadolinium-free alternatives to enhance MRI, especially for targetted renal applications. Manganese exhibits all of the physical qualities that make gadolinium a highly efficient imaging probe in T1-weighted MRI protocols. Both ions are identified by high spin quantum numbers, long longitudinal electronic relaxation times, and fast water exchange kinetics ${ }^{15}$. Further, manganese is biogenic and cleared by biliary excretion when intravenously administered, a particularly attractive feature in the context of renal compromise. Indeed, the complex [Mn (DPDP)] was approved for use as a liver imaging agent ${ }^{16}$.

Nanotechnology has guided the advancement of functional nanomaterials in diverse research areas such as chemistry, engineering, material, and biomedical sciences ${ }^{17}$. Carbon, present in all organic life forms, plays a vital role in developing nanomaterials from three-dimensional graphite, two-dimensional graphene, and graphene oxide to one-dimensional carbon nanotubes. Carbon quantum dots (CQD) is a modern member of the notable family of carbon-based nanomaterials, which was discovered by applying gel electrophoresis to separation and purification ${ }^{18}$ of single-walled carbon nanotubes (SWCNTs). Since 
then, a significant number of further studies have been conducted to explore its luminescent properties ${ }^{19}$. They usually comprise amorphous or nanocrystalline nuclei with a predominance of $\mathrm{sp}^{2}$ hybridized carbon $^{20}$. These carbon-based nanomaterials are small in size $(\leq 10 \mathrm{~nm})$, have near-spherical morphology, chemical inertness, facile functionalization, and multicolor luminescence, and reduced toxicity. Also, exhibit high photostability, resisting photobleaching, and high solubility in aqueous media and organic solvents, which is superior to other fluorescent nanoparticles ${ }^{21}$. These properties are indispensable for applications requiring long term imaging and attracted significant research interest in various fields as a nontoxic alternative to inorganic NPs ${ }^{22}$. All the mentioned features have led to exceptional biological applications in bioimaging, drug release, and theranostic development ${ }^{23}$.

Given the relevance of manganese and the exciting features of CQDs in the field of contrast agent development, the present work consists of designing non-passivated manganese-based carbonaceous NPs (MnCQD) and explore their distribution, toxicological and clearance aspects in Black C57BL/6 mouse model by $\mathrm{T}_{1}$-weighted MRI. Besides, we examined the MnCQD interactions with plasma proteins due to their significance of opsonization to MPS activities. A detailed investigation involving the interaction of the MnCQDs with the two most abundant blood opsonin (BSA and HTF) can provide a piece of evidence for the nanoparticle immunity against MPS.

\section{Results}

\section{CHARACTERIZATION OF THE MANGANESE-BASED NANOPARTICLES.}

\section{Morfologies, size and composition}

The morphologies and the size distributions of the MnCQD systems were investigated from Transmission electron microscopy (TEM) images. Thus, Fig. 1a reveals that MnCQD has almost spherical shapes regularly distributed over the whole surface of the support, and Fig. $\mathbf{1 b}$ reveals an average nanoparticle size of $\approx$ $12 \mathrm{~nm}$. The presence of the Mn was confirmed by analyzing the NP composition provided by the EDS experiments. The EDS spectra (Fig. 1c) confirm the presence of $15.9 \%$ of manganese, $39.1 \%$ of carbon, and $14.6 \%$ of oxygen.

\section{Spectroscopic features}

CQDs show typically strong optical absorption in the UV region $\left(260-320 \mathrm{~nm}\right.$ ) because of $\pi-\pi^{*}$ transition of $\mathrm{C}=\mathrm{C}$ bonds with a tail extending into the visible range. The spectra in Fig. 1d revealed characteristic MnCQD absorption profiles ${ }^{24}$. Absorption spectra have bands centered at $285 \mathrm{~nm}$ that may be associated with an $\mathrm{n}-\pi^{*}$ transition and an absorption band around $260-320 \mathrm{~nm}$, which refers to the $\pi$ - $\pi^{*}$ electronic transitions of $\mathrm{C}=\mathrm{C}$ bonds, aromatic rings in the carbon dot structure, while the absorption range of 350 $-550 \mathrm{~nm}$ is attributed to surface functional groups ${ }^{25}$. The photo-luminescent properties were studied from the emission-excitation spectra shown in Fig. 1e. The fluorescence profile of the MnCQD follows typical carbon dots profile ${ }^{26}$ with a decrease in photoluminescence intensity and redshift when the excitation wavelength increases. The mechanism involved CQDs fluorescence is not yet fully understood and been still a controversial subject in the literature ${ }^{27}$. Some theories have been proposed based on quantum confinement phenomena and radiative recombination of excitations as well as the size-dependent emission of the $\mathrm{CQD}^{28}$.

The infrared spectra are displayed in Fig. 1f. The absorption bands at $3350 \mathrm{~cm}^{-1}$ referring to $\mathrm{OH}$ stretch vibrations, the $\mathrm{C}=\mathrm{O}$ stretch band at $1620 \mathrm{~cm}^{-1}$ (or NH folding) and the bands present at $1092 \mathrm{~cm}^{-1}$ referring to the CO stretch give evidence of the presence of oxygenated functional groups, such as carboxylic acid and 
hydroxyl groups. C-O-H bending was missing, which can also be C-H stretching and deformation (1470$1360 \mathrm{~cm}^{-1}$ ). Note also the bands of vibration absorption of the $\mathrm{C}-\mathrm{N}$ stretch at $1238 \mathrm{~cm}^{-1}$, folding out of the $\mathrm{N}-\mathrm{H}$ plane at $850 \mathrm{~cm}^{-1}$ and $\mathrm{N}-\mathrm{H}$ stretch at $3200 \mathrm{~cm}^{-1}$, indicating the presence of amine groups.

\section{MnCQD display high relaxivities}

Relaxivities are parameters that relate to the relaxation rate of a chemical species as a function of their concentration and is used to measure the performance of the MRI contrast agents. With this goal, MnCQD relaxivities were determined by fitting the experimental relaxation rates of water samples at different concentrations of MnCQD. Fig. $1 \mathrm{~g}$ shows the results obtained of this fitting process, which indicated an excellent linearization of the experimental data to the Eq. 4. The slope of these lines provided the respective MnCQD relaxivities and revealed their high values $\mathrm{r}_{1}=6.96 \mathrm{mM}^{-1} \mathrm{~s}^{-1}$ and $\mathrm{r}_{2}=287.5 \mathrm{mM}^{-1} \mathrm{~s}^{-1}$ and presented similar relaxivities when compared with commercial contrast agents ${ }^{29}$, see Fig. $\mathbf{1 h}$ and Fig. 1i. For instance, commercial agents such as Endorem $\left(\mathrm{r}_{1}=2.8 \mathrm{mM}^{-1} \mathrm{~s}^{-1}\right.$ and $\left.\mathrm{r}_{2}=176 \mathrm{mM}^{-1} \mathrm{~s}^{-1}\right)$, present very comparable relaxivities than the nanoparticles synthesized in our work. These results expose MnCQD as a competitive alternative to gadolinium-based compounds and gadolinium-based nanocomposites ${ }^{30}$.

KidNEY TARGETING AND RENAL CLEARANCE BY T $\mathrm{T}_{1}$ - WEIGHTED MRI.

MRI is a non-invasive method, and it is a complementary technique to follow MnCQD biodistribution, as well as their degradation process. It is well-known that the liver and spleen are initially the most common organ for the accumulation of the majority of untargeted $\mathrm{NPs}^{2}$. Nevertheless, studies involving NP selective targeting of other organs like kidneys are scarce ${ }^{31}$. Fig. 2a shows the NP distribution in different sagittal sections before and after MnCQD mouse intravenously injection. The sagittal sections reveal a significant contrast increase in the kidneys after $7 \mathrm{~min}$, due to the localizing NPs in this organ. As time went on, the contrast continues increasing until the maximal signal has been archived after $3.5 \mathrm{~h}$ of the injection.

A more careful analysis in the MRI images (Fig. 2b) reveals a contrast increase on the mouse bladder at maximal contrast time ( $3.5 \mathrm{~h}$ after the injection), suggesting urine excretion of the NPs. Under a design point of view, MnCQDs is small enough $(<200 \mathrm{~nm})$ to avoid their capture by the spleen but large enough $(\geq 5 \mathrm{~nm}$ ) to prevent kidney elimination resulting from glomerular filtration followed by rapid uptake/elimination into the proximal tubular cells ${ }^{32}$. Even when MnCQD exceeds the filtration size threshold, the renal system clears them. Recognizing physicochemical differences, similar-sized Gd-based CQDs $(\approx$ $12 \mathrm{~nm}$ ) exhibited the equivalent behavior, archiving urine elimination in a very efficient fashion ${ }^{33}$. These pieces of evidence reveal a possible size tolerance for renal excretion of this particle type.

\section{TOXICOLOGICAL ASPECTS}

For toxicological safety, it is crucial to have complete clearance after the MRI analysis. Fig. 2a reveals that contrast signals return to pre-injection levels after $24 \mathrm{~h}$, indicating total particle excretion. A negligible MnCQD quantity maintain localized in the kidney after this time and reveals that the NPs target more specifically the outer stripe of the medulla in the kidneys, matching the proximal straight tubules. It is expected due to the MnCQD reabsorption at this nephron portion. It is also noteworthy that although the mouse histological analysis was not performed at the dose used (15 mg/ $\mathrm{kg}$ animal weight), days after intravenous administration of MnCQD, no external signs of toxicity were observed. Furthermore, the MnCQD biodistribution (Fig. 2c) evidences the high MnCQD selectivity to the kidneys, being almost all NPs localized at this organ.

Cell viability assay is the most frequently investigated parameter to evaluate NP cytotoxicity. The low 
toxicity of CQD has attracted considerable attention and has been investigated by many research groups. CQDs generally displays low toxicity in MTT assays ${ }^{34}$. Here, the viability tests were performed on human lung adenocarcinoma (A549), human Colon adenocarcinoma (HTC-116), and human brain microvascular endothelial (HBMEC) cells. The results are displayed in Fig. 2d, which shows no reduction in the cell viability for all considered cells until the addition of $0.5 \mu \mathrm{M}$ MnCQD, which evidences the very low toxicity of the obtained NPs.

\section{MNCQD AND PLASMA PROTEIN INTERACTIONS}

\section{Fluorescence quenching mechanism}

From the pharmacokinetic point of view, it is very crucial to investigate nanoparticle-plasma proteins since the nanoparticles could potentially impact the structure/function of the proteins. Fluorescence spectroscopy is the most widely used technique for elucidating biochemical interactions with molecules, macromolecules, supramolecular aggregates, and $\mathrm{NPs}^{35}$. It is a simple analytical technique with high sensitivity and is widely used to study structural changes, interactions, and macromolecule stability. The BSA fluorescence quenching has been observed as the MnCQD is titrated to the protein solution (Fig. 3a). It is an unambiguous indication that the MnCQD binds firmly to BSA. Also, the quenching mechanism was determined by analyzing the temperature behavior of the $K_{s v}$. Since the $K_{s v}$ values decrease as the temperature increases for MnCQD-BSA complex (Fig. 3b), the quenching mechanism for MnCQD-BSA complex follow a static mode ${ }^{35}$. The reduction in $\mathrm{K}_{s v}$ values is related to the formation of a non-fluorescent complex. Thus, the increasing temperature seems to decrease the stability of the ground state complex, resulting in a reduction of $\mathrm{K}_{s v}$. Similar results have been observed for the MnCQD-hTF complex.

\section{Affinities and thermodynamic aspects of the interactions}

The affinity of MnCQD to the carrier proteins is a relevant parameter for the development of these particles as contrast agents. That information can be inferred from the association constant values $\mathrm{K}_{a}$ between the CQD and the proteins. From equation 2, the modified Stern-Volmer plots were constructed for the BSAMnCQD (Fig. 3c). The $\mathrm{K}_{a}$ values obtained were in the order of $10^{6} \mathrm{M}^{-1}$ and revealed a high affinity between MnCQD and BSA. Similar results have been observed in other studies involving the interaction of plasma proteins with silver, gold, and zinc NPs ${ }^{36}$. Additionally, MnCQD presents very selective affinity to BSA when compared with hTF (Fig. 3d), or even when compared with similar CQD binding investigation with other proteins reported in the literature ${ }^{37-39}$.

Various types of non-covalent interactions can mediate molecule binding to proteins such as hydrogen bonds, van der Waals, hydrophobic and electrostatic forces. In this context, the main drive forces have been identified by fitting the experimental data to equation 3 and by determining the thermodynamic parameter related to the interaction ${ }^{40}$. The data fitting is shown in Fig. 3e for BSA-MnCQD complex. The values of $\left(\Delta G^{0}\right)$ calculated at the three experimental temperatures were negative, indicating the spontaneity of the MnCQD-BSA formation. In particular, the signals and magnitudes of the $\Delta H^{0}$ and $\Delta S^{0}$ may point to the main force contributing to the interaction. The thermodynamic parameter values obtained for the complexes BSA-MnCQD and HTF-MnCQD (in parenthesis). It can be noted that the determined $\Delta H^{0}=-$ 79.29(-63.35) $\mathrm{Jmol}^{-1}$ are negative and $\Delta S^{0}=+378.78(+293.98) \mathrm{kJmol}^{-1} \mathrm{~K}^{-1}$ are positive for both complexes. These values indicate that electrostatic forces and hydrogen bonds are the main drive forces responsible for the formation of the complexes ${ }^{41}$. 


\section{Structural changes in the protein-nanoparticle complexes.}

Circular dichroism is a powerful tool that allows the analysis of secondary protein structure and also the study of protein interactions with other molecules. In this study, we recorded BSA CD spectra in the presence or absence of the MnCQD, and the results are displayed in Fig. 3f. In the absence of MnCQD, we observed typical protein CD spectra for both proteins, exhibiting negative peaks at 208 and $222 \mathrm{~nm}$, which is characteristic of the secondary structure of the proteins ( $\alpha$-helix, $\beta$-sheet and turns). The peak intensities reduced as the MnCQD are added to protein solutions, indicate a loss of protein secondary structure in both formed complexes.

MnCQD opsonization has been examined by incubating the NPs with BSA, Fetal Bovine Serum (FBS) and Mouse Serum (MS). Following the incubation, alterations in the effective diameter (Fig. 3g) and zeta potential (Fig. 3h) of the formed supramolecular structures have been monitored. As can be observed in Fig. 3g, the effective diameter of the MnCQD supramolecular structures formed by binding to BSA (30.3$47.6 \mathrm{~nm})$ and FBS proteins $(19.3-32.3 \mathrm{~nm})$ are smaller than the structures formed in water dispersion (90.1$104.2 \mathrm{~nm}$ ). On one side, The peak in the range of 5.3-6.9 nm might be attributed to free smaller NPs displayed in the distribution of the Fig. 1b, which implies that smaller components possess fewer capabilities to grow supramolecular structures due to their reduced superficial area. On another side, the highest diameter NPs in the size distribution has a larger surface area to form supramolecular structures with FBS, which could be linked to the peak observed in the range of $74.9-76.4 \mathrm{~nm}$ (Fig. 3f). These results are indicating a negligible level of the MnCQD opsonization by both bovine proteic media. Also, very supporting results emerge from the zeta potential analysis of the MnCQD supramolecular structures(Fig. $3 \mathbf{h}$ ). According to the zeta potential value of $-9.4 \pm 1.0 \mathrm{mV}$ for MnCQD in aqueous media, it can be perceived that the NPs are good dispersible and stable for a very long time in the water, with no signs of flocculation or precipitation. Gains in MnCQD colloidal stability have been observed in the BSA $(\zeta=-13.9 \pm 0.9 \mathrm{mV})$ media compared with aqueous media. Notwithstanding, more substantial NP stability gains are observed in the FBS $(\zeta=-$ $26.2 \pm 1.3 \mathrm{mV})$ and $\mathrm{MS}(\zeta=-23.2 \pm 2.0 \mathrm{mV})$ concerning water media. Similar zeta potential values for FBS and MS also imply low-level opsonization of the MnCQD in MS media and might explain the non-elimination of the MnCQD by the MPS system.

\section{Discussions}

MnCQD relaxivities are similar in magnitude to Gadolinium-based complexes, and the critical difference is linked to the metal distribution. If MnCQD undergoes degradation occasioning Mn liberation, an efficient endogenous elimination pathway will occur through the hepatobiliary system. Such a path does not exist for gadolinium, which can lead to Gd accumulation and toxicity ${ }^{42}$. One of the first attempts to solve Gd toxic concerns applying carbonaceous nanomaterials was based on designing nanocomposite Gd@Cdots ${ }^{33}$. Although the reported nanocomposite exhibited excellent features, safety concerns are still an open question for gadolinium-based nanomaterials.

Although MnCQD is not explicitly designed to target the kidneys, the NP selectivity to this organ is unexpected but highly desirable for diagnosis purposes. Although MnCQDs were targeting the kidneys efficiently without any stealth agent, insinuating absolute protection from the MPS. Since the correlation between NP physicochemical properties and biodistribution is not fully understood, several studies expose (in a molecular size basis) unexpected renal selectivity and clearance by non-passivated NPs ${ }^{43}$.

Another important observation displayed in Fig. 3a is related to the maximal fluorescence peak $\left(\lambda_{\max }\right)$. BSA is well-known to have only two fluorescent tryptophans localized in the Sudlow site I and II, while 
site III has no fluorescent residue. During the BSA quenching by MnCQD (Fig. 3a), no changes in $\lambda_{\max }$ have been observed. It reveals protein structure stability around the fluorescent residues, e.g., more concretely sites I and II, during BSA-MnCQD interaction. Furthermore, a recent reporter investigated the displacement of the well-known site ligand (warfarin and ibuprofen) and identified sites I and II of human serum albumin as the anchoring region for the CQD nanoparticles ${ }^{38}$. HTF experiences a visible shift on the $\lambda_{\max }=338 \mathrm{~nm}$ to $345 \mathrm{~nm}$ (Fig. $3 \mathbf{b}$ ), which indicates a not trivial protein structural changes.

Due to reported BSA site I and II stability, it suggests that the secondary structure loss may occur onsite III, and the impact of these site structural changes on the organ preferences is not evident. A more intuitive role on the subject can be established by analyzing the transport features of well-known protein and peptides. Low Molecular Weight Proteins (LMWP) have been extensively used as drug carrier targetting kidneys, despite LMWP negative impact on renal toxicity, immune refusal, and systemic blood pressure ${ }^{44}$. Alternatively, some studies investigate albumin fragments to the task ${ }^{45}$, being recognized peptide fragment PF299-585 as the most promising protein part capable of preferential accumulation in the renal tubules over the glomeruli (around 40\% retention). Interestingly, the low structured site III (comprising residues 384-585) in the complex BSA-MnCQD has a significant portion of the most relevant kidney targetting albumin fragment ( PF299-585). It implies that site III plays a crucial role in selectively transporting the complex BSA-MnCQD to the organ.

Moreover, protein structural modification on the HTF-MnCQD complex is corroborated by $\lambda_{\text {max }}$ shifts observed on the fluorescence quenching experiments. Our previous investigation ${ }^{46}$ with small anionic non-metallic CQDs from the same source revealed that the HTF also underwent secondary structure modification through NP interaction, and it was followed by subtle iron release from HTF. Despite these relevant observations, it was still not possible to identify the modified protein region by complex formation with CQDs and also with MnCQDs.

The use of metal ion encapsulated carbon NPs as a contrast agent for MRI offers many advantages. They may be presented as a hopeful alternative in the diagnosis and treatment of diseases, as well as being non-toxic and having high specificity for the kidneys, as presented in vivo experiments. The use of nanostructures would allow visualization of minimally-sized MRI lesions, enabling early diagnosis. MnCQD has pharmacokinetic and pharmacodynamic analogous behavior to gadolinium complexes (produces positive image contrast) without the demand for any bioconjugation to reduce the administered dose or increase sensitivity. In summary, we have successfully synthesized and characterized manganese-based carbon nanoparticles (MnCQD) with excellent MRI/fluorescence behaviors by a simple one-step synthesis strategy. The MnCQD provided a high affinity to the renal system for targeted imaging. In vitro and in vivo results demonstrate that the probes have excellent biocompatibility, inconspicuous in vivo toxicity, and efficient excretion through renal clearance. Moreover, the MnCQD combination of extraordinary fluorescence and high relaxivities remodels them as promising MRI/fluorescence bi-functional imaging probes for an accurate diagnosis.

\section{Methods}

Transmission electron microscopy (TEM) MnCQD was analyzed on the FEI Tecnai Spirit iCorr $120 \mathrm{Kv}$ equipment. The powdered nanoparticles placed in $1.5 \mathrm{ml}$ eppendorf tubes were dissolved in water and dispersed in an ultrasonic bath USC - $750 \mathrm{~A}, 25 \mathrm{kHz}$ for 30 minutes. The preparation of the grids was performed by dripping the MnCQD aqueous solution onto the surface of the carbon-coated copper grid, followed by drying for three hours at room temperature before the examination. The presented Energy Dispersive X- 
Ray Spectroscopy (EDS) results have been performed using a scanning electron microscope model MIRA3 TESCAN, with a resolution of $1 \mathrm{~nm}$ depending on the sample.

Dynamic Light Scattering (DLS) and Zeta potential The effective diameter and polydispersion of NP samples were measured using Quasi-Elastic Light Scattering (QELS) in a OMNI Particle Sizing analyzer (Brookhaven Instruments Corporation, Holtsville, NY), as described by Frases et al. ${ }^{47}$. Measurements were taken at $25^{\circ} \mathrm{C}$. The multimodal distributions of particle diameter size were analyzed using algorithms that calculate the non-negative least squares (Non-Negatively Constrained Least Squares algorithm - NNLS) based on the dispersion of the light intensity given by each particle. All samples were analyzed under the same conditions. The Zeta potential and the mobility of NP samples were analyzed at ZetaPALS (Brookhaven Instruments Corp, Holtsville, NY), as described by Frases et al. ${ }^{47}$. Measurements were made at $25^{\circ} \mathrm{C}$.

Photoluminescence experiments Emission luminescence spectroscopic measures were recorded at 298 K on a Jobin-Yvon Raman or a U-1000 dual-monochromatic equipment. Excitation of 1.1 and $2.2 \mathrm{~mm}$ and emission of $610 \mu \mathrm{m}$ were used.

UV-Visible (UV-Vis) absorption measurements: The light absorption spectrum in the visible ultraviolet (UV-visible) region of the MnCQD was recorded on a UV-Vis-NIR spectrophotometer (Erkin Elmer-lambda 650) using a $1 \mathrm{~cm}$ cuvette.

Fourier-transform infrared spectroscopy (FTIR): FTIR spectra were recorded on an IR tracer-100 Shimadzu infrared spectrophotometer operating in the frequency range of $500-4000 \mathrm{~cm}^{-1}$, using $\mathrm{KBr}$ pellets as the sample matrix.

Fluorescence quenching experiments Protein fluorescence quenching experiments were performed on a Cary Eclipse fluorescence spectrometer (Varian, Sydney, Australia). Slot widths of $10 \mathrm{~nm}$ were used for excitation and emission, and temperatures were adjusted to 293, 298 and $303 \mathrm{~K}$. The $\mathrm{K}_{s v}$ constants for the interaction curves, which corresponds to MnCQD-BSA and MnCQD -HTF complexes, were calculated by the Stern-Volmer equation ${ }^{35}$

$$
\frac{F_{0}}{F}=1+K_{s v}[Q]
$$

Where $\mathrm{F}_{0}$ and $\mathrm{F}$ are the fluorescence intensities of proteins in the absence and presence of MnCQD, respectively, and [Q] is the concentration of nanoparticles MnCQD. The MnCQD affinities to proteins were obtained by the modified Stern-Volmer equation

$$
\log \left[\frac{F_{0}-F}{F}\right]=\log K_{a}+n \log [Q]
$$

$\mathrm{K}_{a}$ is the association constant, $\mathrm{n}$ is the number of binding sites. To calculate the thermodynamic parameter involved in the interaction of MnCQD with BSA and HTF, we used the Van't Hoff equation

$$
\ln K_{a}=\left(-\frac{\Delta H}{R}\right) \frac{1}{T}+\left(\frac{\Delta S}{R}\right)
$$

Where $\mathrm{K}_{a}$ is the association constant at each temperature; $\mathrm{R}$ is the gas constant $\left(8.314 \mathrm{Jmol}^{-1} \mathrm{~K}^{-1}\right)$, T is the temperature in Kelvin, $\Delta \mathrm{H}$ is the enthalpy variation and $\Delta \mathrm{S}$ is the entropy variation. The graph $\operatorname{Ln} K_{a}$ versus $1 / \mathrm{T}$ has as its angular coefficient $(-\Delta \mathrm{H} / \mathrm{R})$ and the linear coefficient $(\Delta \mathrm{S} / \mathrm{R})$. 
Circular dichroism (CD) experiments Protein CD spectra were recorded at each concentration of MnCQD on a Chirascan spectropolarimeter (Applied Photophysics, London, UK) at $298 \mathrm{~K}$ using a $0.01 \mathrm{~cm}$ optical path quartz cuvette. Final spectra were the average of three scans obtained at $190 \mathrm{~nm}$ and $260 \mathrm{~nm}$ wavelengths at a $25 \mathrm{~nm} / \mathrm{min}$ speed after subtracting the spectra corresponding to the baselines and solution.

MTT assay The cytotoxicity of MnCQD was evaluated by the MTT method. HBMEC, A549, and HCT-116 cells were pre-cultured and plated in 96 -well plates (20,000 cells/well). After $24 \mathrm{~h}$, the cells were incubated with MnCQD $(0.00-2 \mathrm{mg} / \mathrm{ml})$ in an oven at $37^{\circ} \mathrm{C}$ and under $5 \% \mathrm{CO}_{2}$ atmosphere for 24 hours. After 20 hours of treatment, $20 \mu \mathrm{L}$ of MTT $(2.5 \mathrm{mg} / \mathrm{mL})$ was added to the wells. The ELISA microplate was further incubated for 4 hours for MTT metabolism. Subsequently, the medium was discarded, and the crystals formed in the cells were solubilized in $100 \mu \mathrm{L}$ of DMSO. Absorbance reading was performed on a plate reader (Molecular Devices - SpectraMax Microplate Reader) at $570 \mathrm{~nm}$ and $650 \mathrm{~nm}$. The cytotoxicity of the MnCQD was calculated as a function of the control, considered as $100 \%$ cell viability.

Relaxivity measurements and in vivo MRI evaluation The $r_{1}$ and $r_{2}$ measurements of the MnCQD was performed in deuterated water on a Varian model Plus Spectrophotometer (Santa Clara, CA, USA) operating at $400 \mathrm{MHz}$. Evaluation of MnCQD suspensions as contrast agents was done by measuring the relaxation times $T_{1}$ and $T_{2}$ at different MnCQD concentration. Since a contrast agent may affect the two relaxation rates $\left(R_{1}=1 / T_{1}\right.$ and $\left.R_{2}=1 / T_{2}\right)$ individually, the two corresponding relaxivities (i=1,2) can be determined by using the equation

$$
\frac{1}{T_{i}}=\frac{1}{T_{i}^{0}}+r_{i}[N P]
$$

Where $T_{i}^{0}$ is the relaxation times in the absence of the nanoparticles and [NP] is MnCQD concentration.

The in vivo MnCQD contrast effect was investigated on a 7T 210 Bore Actively Screened Refrigerated Magnet System dedicated to small animals (Varian Inc., Palo Alto, CA). The system is equipped with highperformance gradients and square coils with a $38 \mathrm{~mm}$ diameter and 16 channels. MnCQD was injected intravenously in the male Black C57BL / 6 mice at a dose of $(15 \mathrm{mg} / \mathrm{kg})$. The in vivo evaluation of MnCQD was performed by $\mathrm{T}_{1}$-weighted imaging and were acquired at different pre and post time intervals at 7,15 , 30, 45, 60min, 3h, and $24 \mathrm{~h}$ after intravenous injection.

\section{Acknowledgements}

Authors are thankful to CNPq (Conselho Nacional de Desenvolvimento Científico e Tecnológico, Brazil) for financial support (Grant number: 420902/2016-3), CAPES (Coordenação de Aperfeiçoamento de Pessoal de Nível Superior, Brazil), FACEPE (Grant APQ-0459-1.06/2015) and FAPERJ (Fundação Carlos Chagas Filho de Amparo a Pesquisa do Estado do Rio de Janeiro).

\section{References}

[1] E. F. Simões, J. C. E. da Silva, J. M. Leitão, Analytica chimica acta 2014, 852174.

[2] J. P. M. Almeida, A. L. Chen, A. Foster, R. Drezek, Nanomedicine 2011, 6, 5815.

[3] L. T. M. Sa, M. de Souza Albernaz, B. F. de Carvalho Patricio, M. V. F. Junior, B. F. Coelho, A. Bordim, J. C. Almeida, R. Santos-Oliveira, Journal of pharmaceutical and biomedical analysis 2012, 70602.

[4] N. Duzgunes, Nanomedicine: Infectious Diseases, Immunotherapy, Diagnostics, Antifibrotics, Toxicology and Gene Medicine, Academic Press, 2012.

[5] H. H. Gustafson, D. Holt-Casper, D. W. Grainger, H. Ghandehari, Nano today 2015, 10, 4487.

[6] T. U. Wani, S. N. Raza, N. A. Khan, Polymer Bulletin 2019, 1-25. 
[7] C. C. Fleischer, C. K. Payne, Accounts of chemical research 2014, 47, 82651.

[8] D. V. Hingorani, A. S. Bernstein, M. D. Pagel, Contrast media \& molecular imaging 2015, 10, 4245.

[9] B. Bonnemain, Journal of drug targeting 1998, 6, 3167.

[10] F. L. Giesel, A. Mehndiratta, M. Essig, European radiology 2010, 20, 102461.

[11] J. L. Major, T. J. Meade, Accounts of chemical research 2009, 42, 7893.

[12] J. Estelrich, M. J. Sánchez-Martín, M. A. Busquets, International journal of nanomedicine 2015, 10 1727.

[13] M. Rogosnitzky, S. Branch, Biometals 2016, 29, 3365.

[14] E. Kanal, M. F. Tweedle, Radiology 2015, 275, 3630.

[15] E. Boros, E. M. Gale, P. Caravan, Dalton transactions 2015, 44, 114804.

[16] D. Zhu, F. Liu, L. Ma, D. Liu, Z. Wang, International journal of molecular sciences 2013, 14, 510591.

[17] Z. Peng, X. Han, S. Li, A. O. Al-Youbi, A. S. Bashammakh, M. S. El-Shahawi, R. M. Leblanc, Coordination Chemistry Reviews 2017, 343256.

[18] X. Xu, R. Ray, Y. Gu, H. J. Ploehn, L. Gearheart, K. Raker, W. A. Scrivens, Journal of the American Chemical Society 2004, 126, 4012736.

[19] S. Zhu, Y. Song, X. Zhao, J. Shao, J. Zhang, B. Yang, Nano research 2015, 8, 2355.

[20] S. Ray, A. Saha, N. R. Jana, R. Sarkar, The Journal of Physical Chemistry C 2009, 113, 4318546.

[21] S. N. Baker, G. A. Baker, Angewandte Chemie International Edition 2010, 49, 386726.

[22] C. Jiang, H. Wu, X. Song, X. Ma, J. Wang, M. Tan, Talanta 2014, 12768.

[23] X. T. Zheng, A. Ananthanarayanan, K. Q. Luo, P. Chen, Small 2015, 11, 141620.

[24] Z. Zhang, J. Hao, J. Zhang, B. Zhang, J. Tang, Rsc Advances 2012, 2, 238599.

[25] W. Du, X. Xu, H. Hao, R. Liu, D. Zhang, F. Gao, Q. Lu, Science China Chemistry 2015, 58, 5863.

[26] H. Wang, J. Shen, Y. Li, Z. Wei, G. Cao, Z. Gai, K. Hong, P. Banerjee, S. Zhou, Biomaterials Science 2014, 2, 6915 .

[27] K. Wang, Z. Gao, G. Gao, Y. Wo, Y. Wang, G. Shen, D. Cui, Nanoscale research letters 2013, 8, 1122.

[28] M. Zhang, H. Ju, L. Zhang, M. Sun, Z. Zhou, Z. Dai, L. Zhang, A. Gong, C. Wu, F. Du, International journal of nanomedicine 2015, 106943.

[29] M. Rohrer, H. Bauer, J. Mintorovitch, M. Requardt, H.-J. Weinmann, Investigative radiology 2005, 40, 11715.

[30] S.-T. Yang, X. Wang, H. Wang, F. Lu, P. G. Luo, L. Cao, M. J. Meziani, J.-H. Liu, Y. Liu, M. Chen, et al., The Journal of Physical Chemistry C 2009, 113, 4218110.

[31] J. E. Zuckerman, C. H. J. Choi, H. Han, M. E. Davis, Proceedings of the National Academy of Sciences 2012, 109, 83137.

[32] Q. Wang, C. Zhang, G. Shen, H. Liu, H. Fu, D. Cui, Journal of nanobiotechnology 2014, 12, 158.

[33] H. Chen, G. D. Wang, W. Tang, T. Todd, Z. Zhen, C. Tsang, K. Hekmatyar, T. Cowger, R. B. Hubbard, W. Zhang, et al., Advanced Materials 2014, 26, 396761.

[34] A. Mohammadi, A. Aliakbarzadeh Karimi, H. Fallah Moafi, Progress in Color, Colorants and Coatings 2016, 9, 4249.

[35] J. R. Lakowicz, Principles of fluorescence spectroscopy, Springer Science \& Business Media, 2013.

[36] A. Albanese, C. D. Walkey, J. B. Olsen, H. Guo, A. Emili, W. C. Chan, Acs Nano 2014, 8, 65515.

[37] S. K. Vaishnav, I. Karbhal, M. L. Satnami, K. K. Ghosh, et al., Journal of Molecular Liquids 2018, 255 279.

[38] Y. Song, H. Wang, L. Zhang, B. Lai, K. Liu, M. Tan, Food \& Function 2020, 11, 32358. 
[39] Z.-Q. Xu, Q.-Q. Yang, J.-Y. Lan, J.-Q. Zhang, W. Peng, J.-C. Jin, F.-L. Jiang, Y. Liu, Journal of hazardous materials 2016, 301242.

[40] C. D. Walkey, W. C. Chan, Chemical Society Reviews 2012, 41, 72780.

[41] X. Zhang, J. Zhang, F. Zhang, S. Yu, Nanoscale 2017, 9, 144787.

[42] A. B. Bourlinos, A. Bakandritsos, A. Kouloumpis, D. Gournis, M. Krysmann, E. P. Giannelis, K. Polakova, K. Safarova, K. Hola, R. Zboril, Journal of Materials Chemistry 2012, 22, 4423327.

[43] R. M. Williams, J. Shah, B. D. Ng, D. R. Minton, L. J. Gudas, C. Y. Park, D. A. Heller, Nano letters 2015, $15,42358$.

[44] R. F. G. Haverdings, M. Haas, A. R. Greupink, P. M. deVries, F. Moolenaar, D. de Zeeuw, D. K. Meijer, Renal failure 2001, 23, 3-4 397.

[45] C.-P. Liu, Y. Hu, J.-C. Lin, H.-L. Fu, L. Y. Lim, Z.-X. Yuan, Medicinal research reviews 2019, 39, 2561.

[46] Y. S. Garcia, M. R. Barros, G. T. Ventura, R. M. de Queiroz, A. R. Todeschini, J. L. Neves, Journal of Molecular Liquids 2019, 292111460.

[47] S. Frases, L. Nimrichter, N. B. Viana, A. Nakouzi, A. Casadevall, Eukaryotic cell 2008, 7, 2319. 


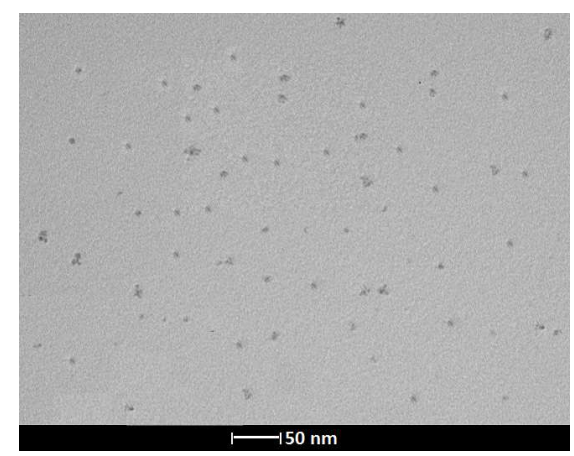

(a)

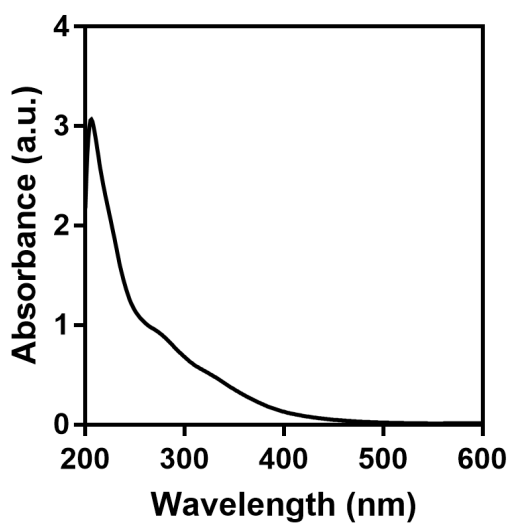

(d)

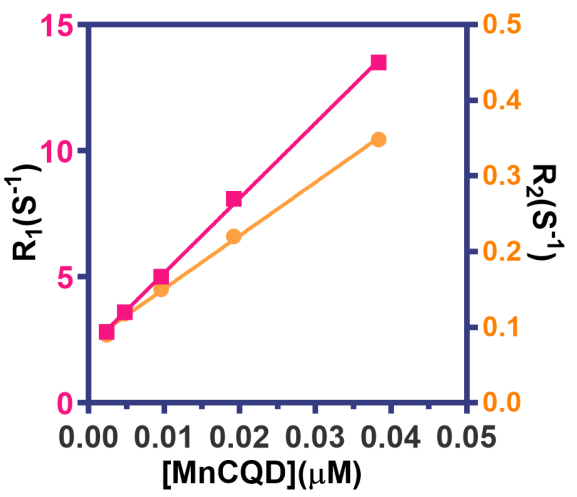

(g)

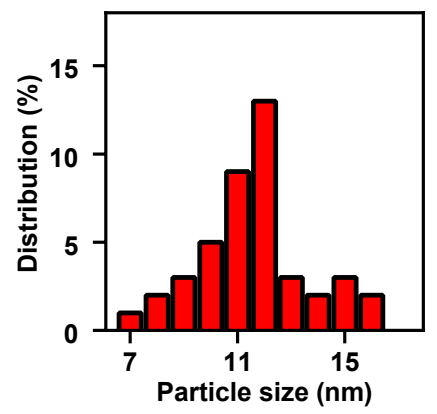

(b)

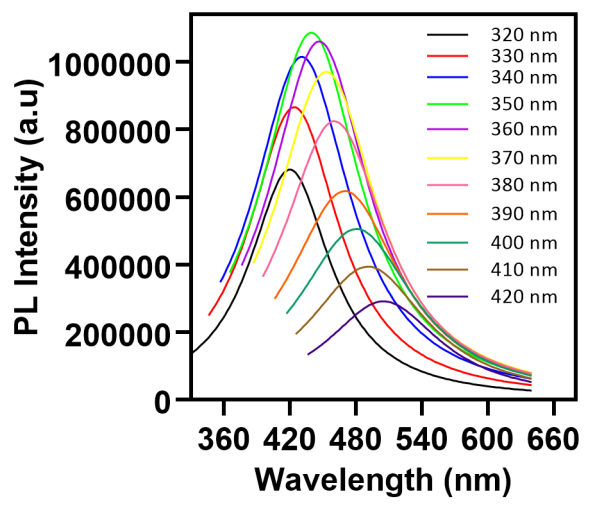

(e)

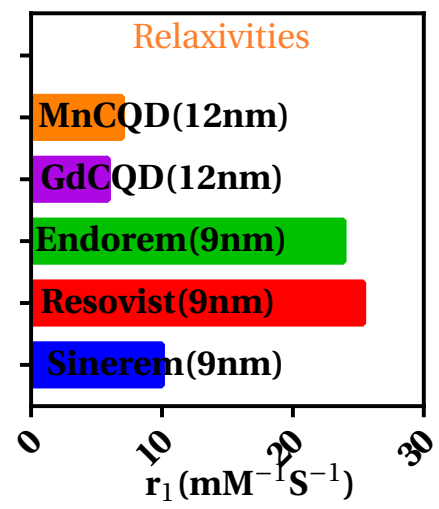

(h)

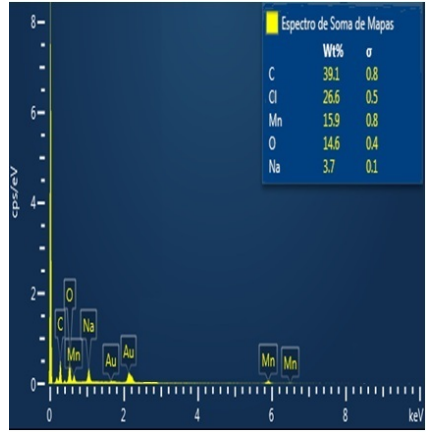

(c)

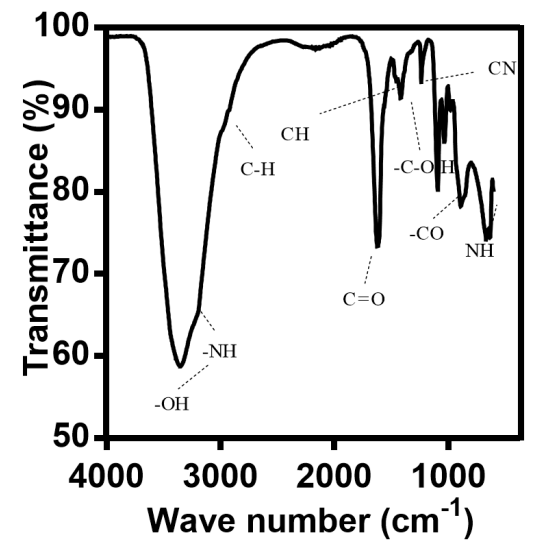

(f)

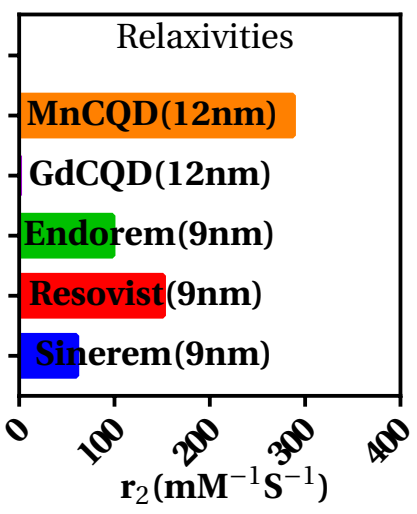

(i)

Figure 1: Chemical, microscopic and spectroscopic characterization of the MnCQD. TEM images (a) displaying the spherical morfologies of the nanoparticles and the size distribution (b). Chemical composition determined by Dispersive energy spectroscopy (c), UV-vis absorption spectra (d), photoluminescence profile (e), and Infrared spectra (f) of the MnCQD. Determination of the relaxivities $r_{1}$ and $r_{2}$ (g) at $298 \mathrm{~K}$ by fitting the experimental data to Eq. 4. Comparison of the MnCQD $r_{1}$ (h) and $r_{2}$ (i) with comercial available contrast agent, revealing comparable $\mathrm{r}_{1}$ and $\mathrm{r}_{2}$ in relation to comercial ones. 


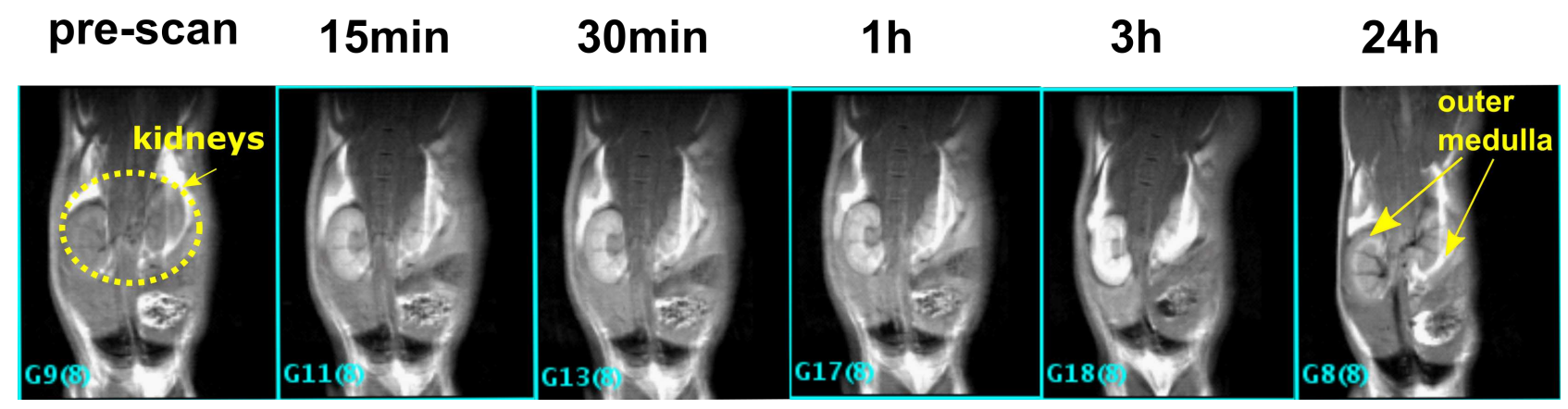

(a)

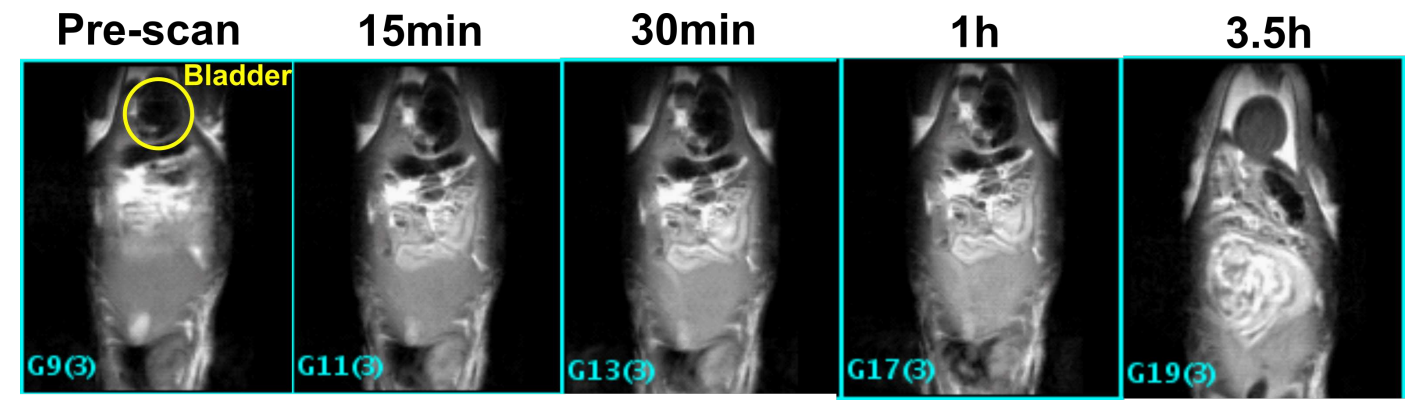

(b)

Temporal Evaluation (Gaussian)

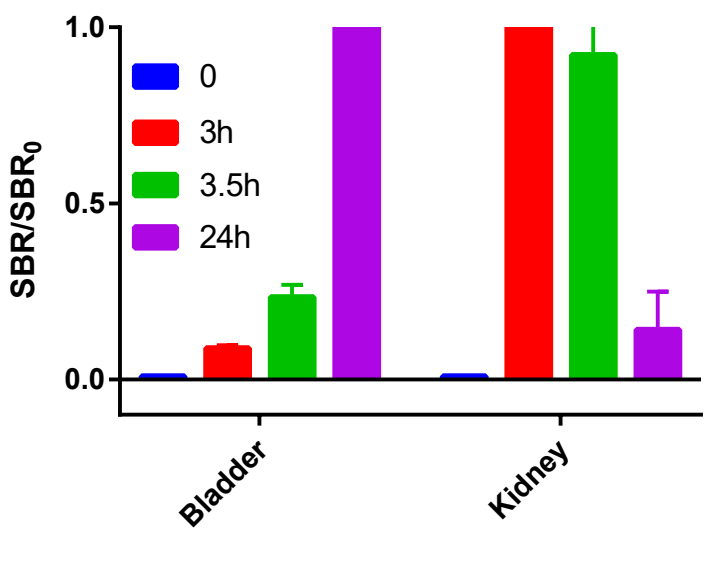

(c)

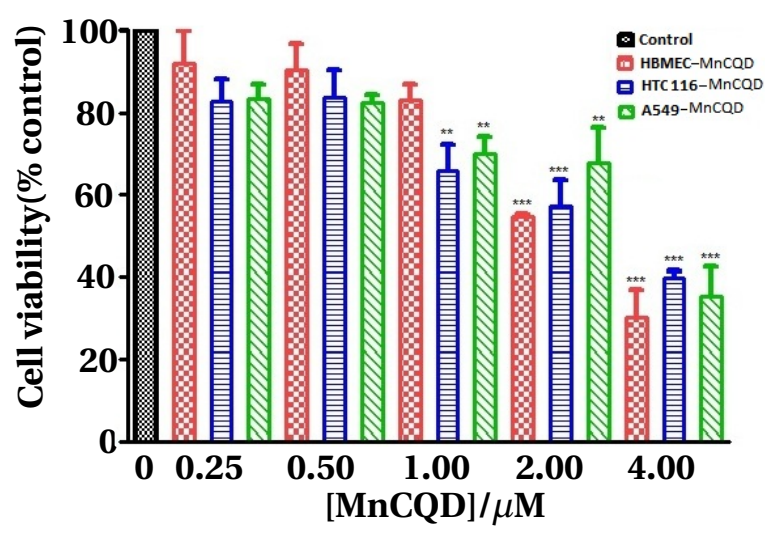

(d)

Figure 2: In vivo evaluation of MnCQD as contrast agent showing the region of the kidneys (a) and the bladder (b). Biodistribution of the nanoparticles (c) in different mouse organ and cell viability assay (d) of MnCQD against the Human Brain Microvascular Endothelial cell (HBMEC), adenocarcinomic human alveolar basal epithelial cell (A549) and human colon cancer cell (HTC116) lines. 


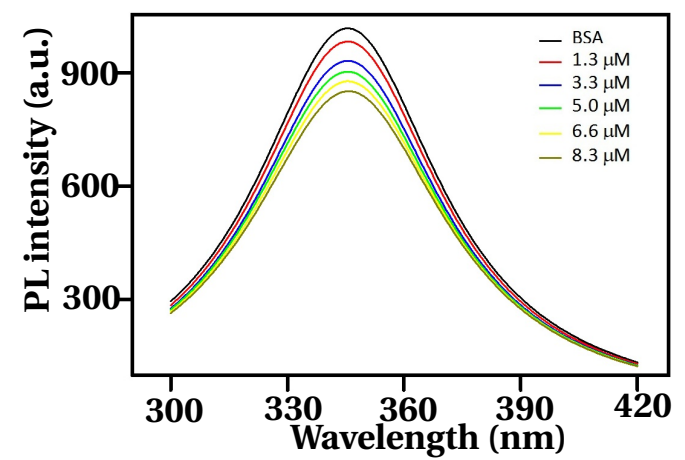

(a)

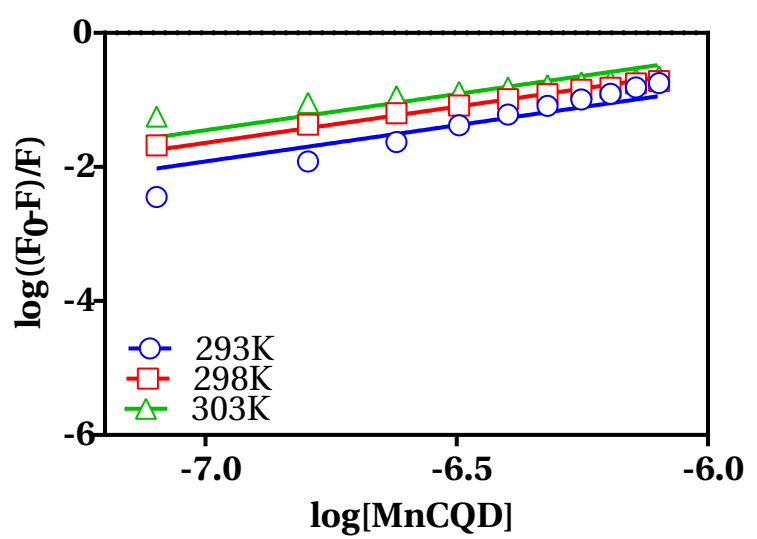

(c)

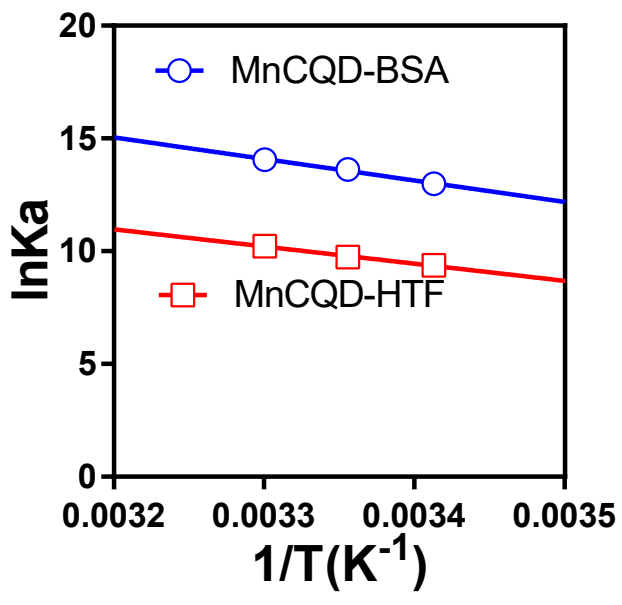

(e)

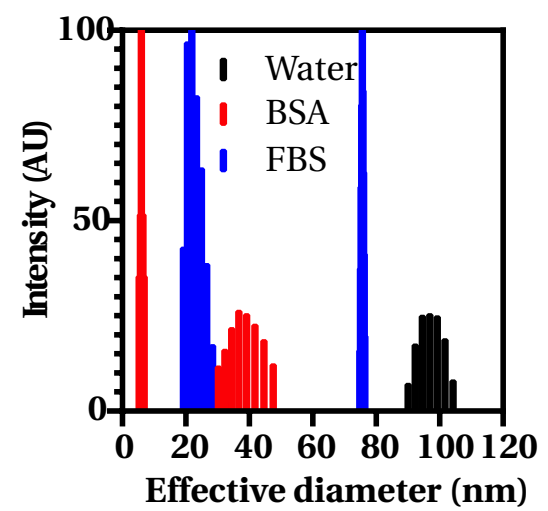

(g)

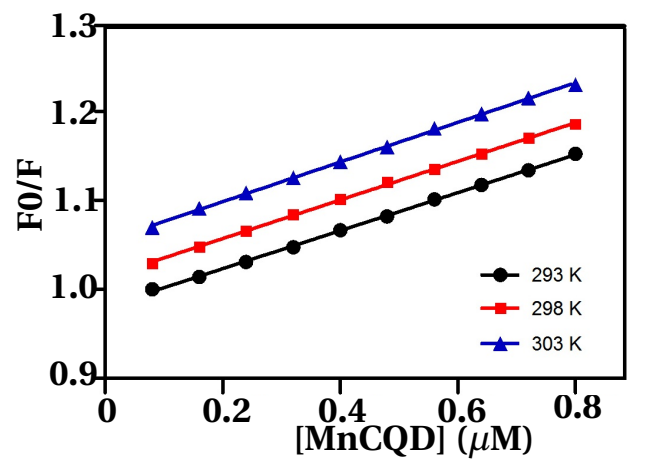

(b)

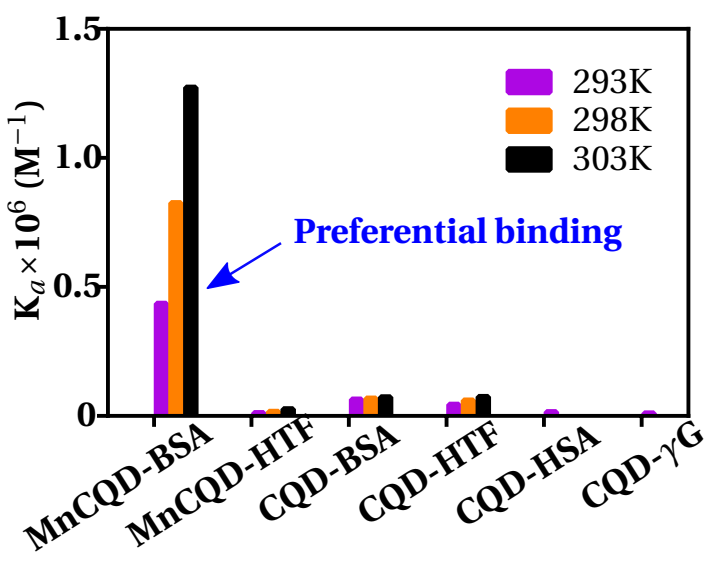

(d)

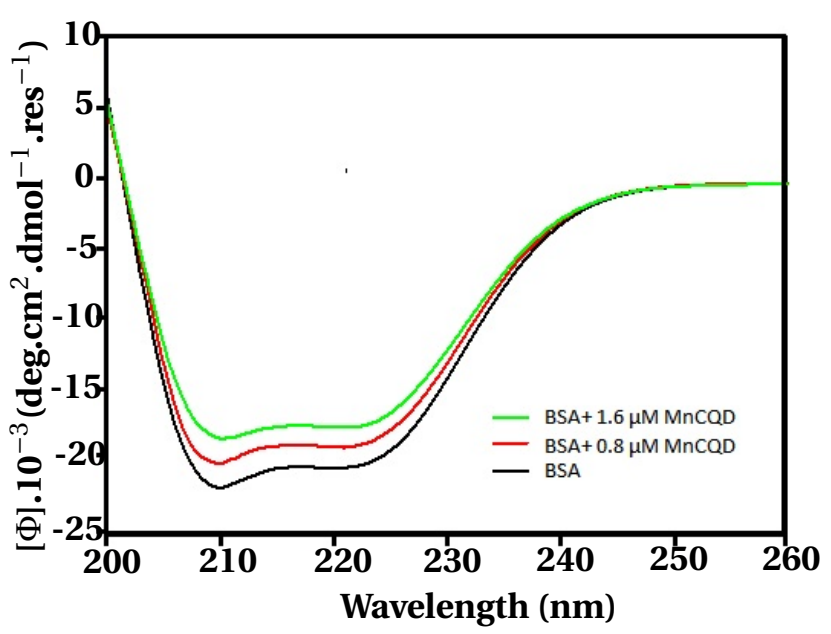

(f)

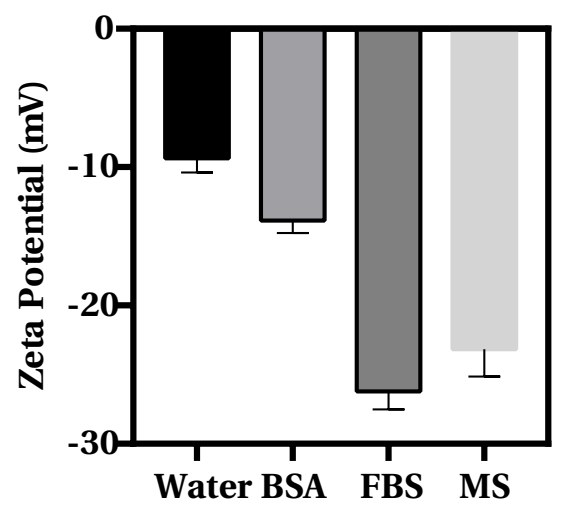

(h)

Figure 3: Fluorescence quenching experiments showing the fluorescence suppression of BSA (a) as the MnCQD are titrated to the proteins. Stern-Volmer plots (b), log plots (c) at three different temperatures, afinities comparison (d), Van't Hoff plots (e) used to determine the thermodynamic parameter. CD spectra of BSA-Mq4CQD complexes (f), DLS spectra (g) and zeta potential (h) of the MnCQD in water, BSA, FBS and MS. 


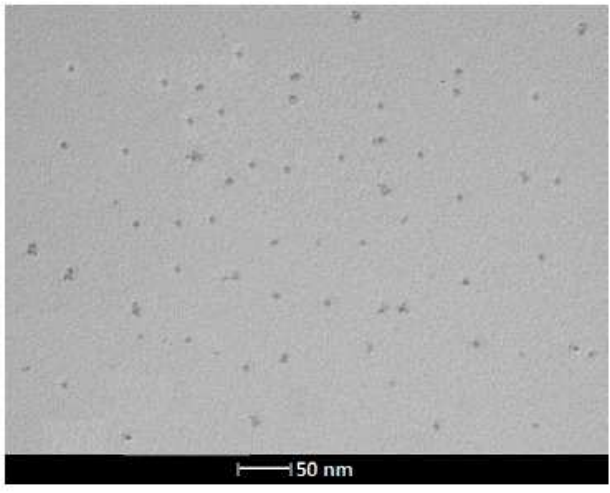

(a)

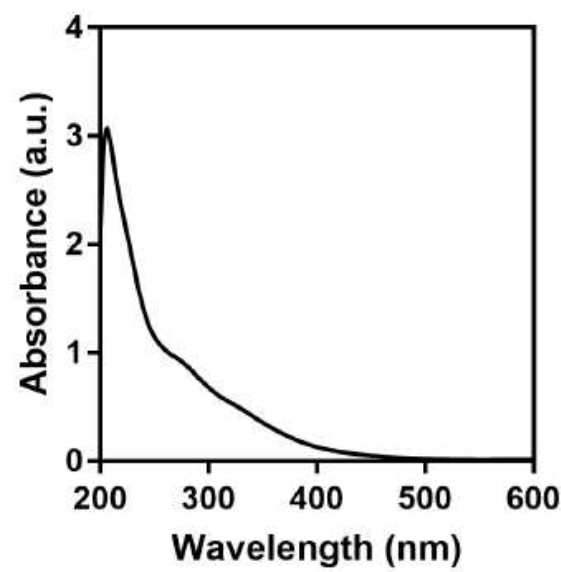

(d)

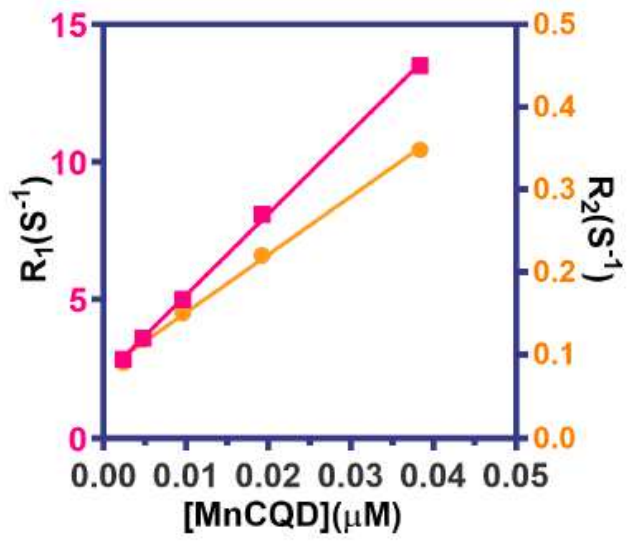

(g)

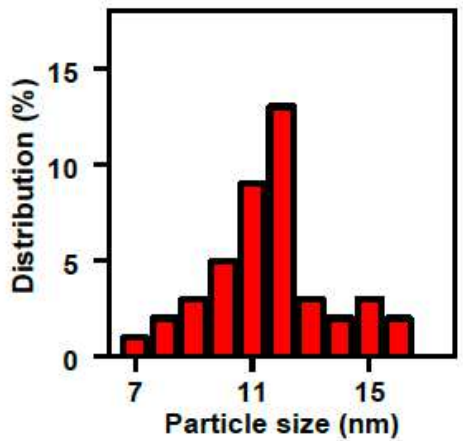

(b)

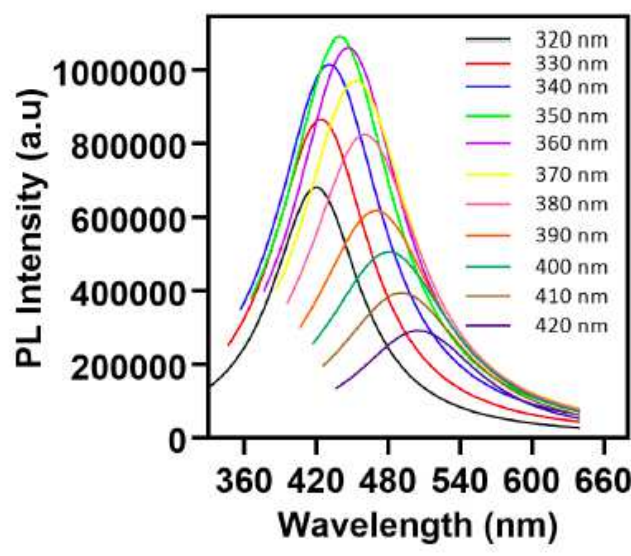

(e)

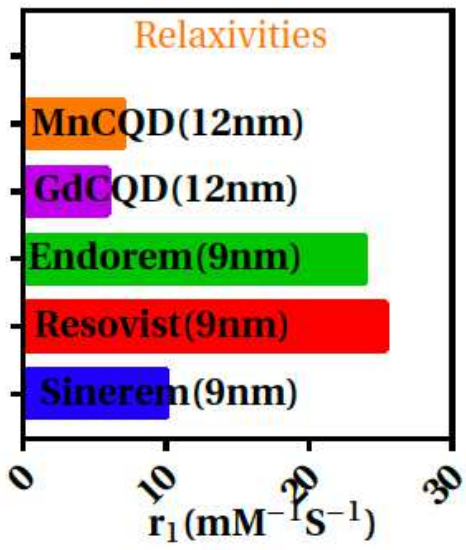

(h)

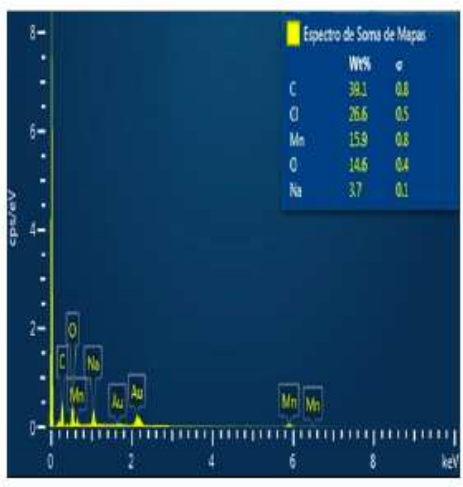

(c)

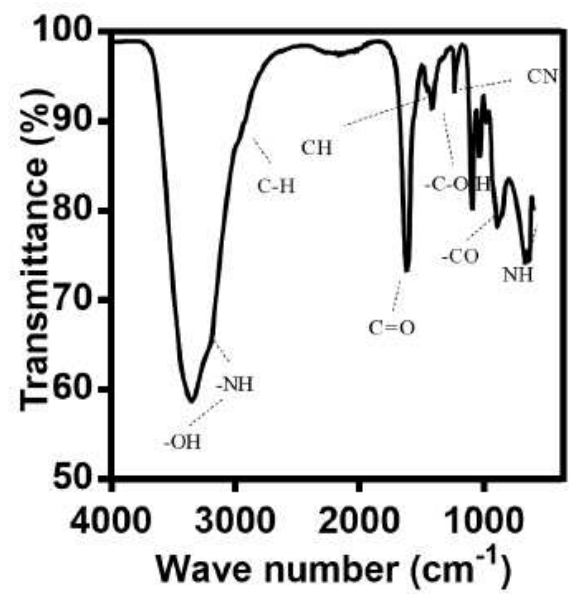

(f)

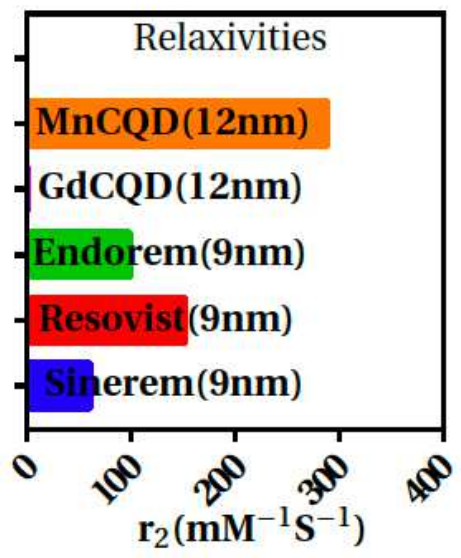

(i)

Figure 1

Chemical, microscopic and spectroscopic characterization of theMnCQD. TEM images (a) displaying the spherical morfologies of the nanoparticles and the size distribution (b). Chemical composition determined by Dispersive energy spectroscopy (c), UV-vis absorption spectra (d), photoluminescence profile (e), and Infrared spectra (f ) of the MnCQD. Determination of the relaxivities $r 1$ and $r 2(\mathrm{~g})$ at $298 \mathrm{~K}$ 
by fitting the experimental data to Eq. 4. Comparison of theMnCQD r1 (h) and r2(i) with comercial available contrast agent, revealing comparable $\mathrm{r} 1$ and $\mathrm{r} 2$ in relation to comercial ones.

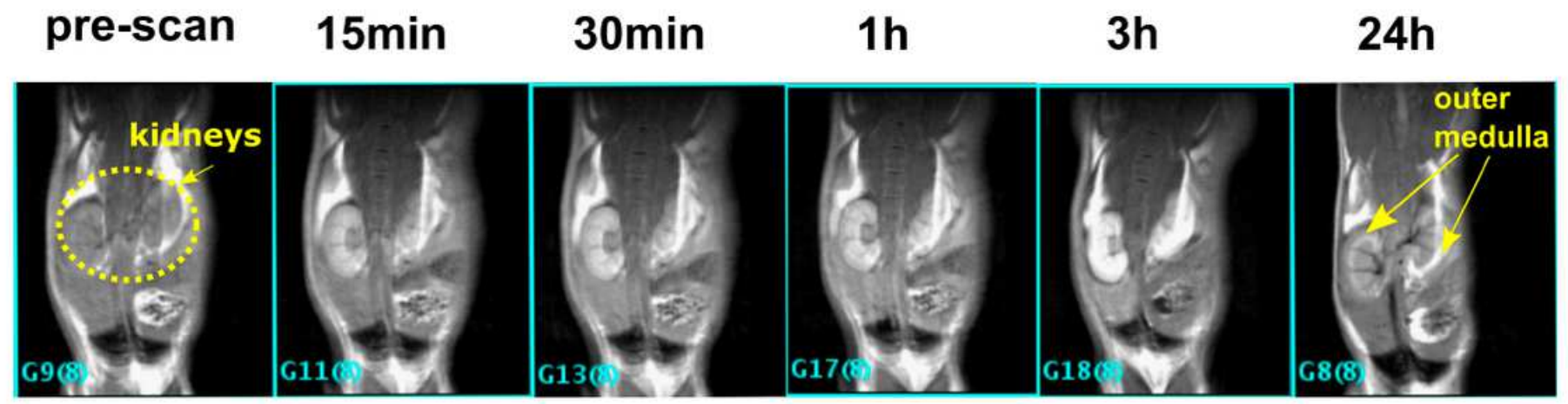

(a)

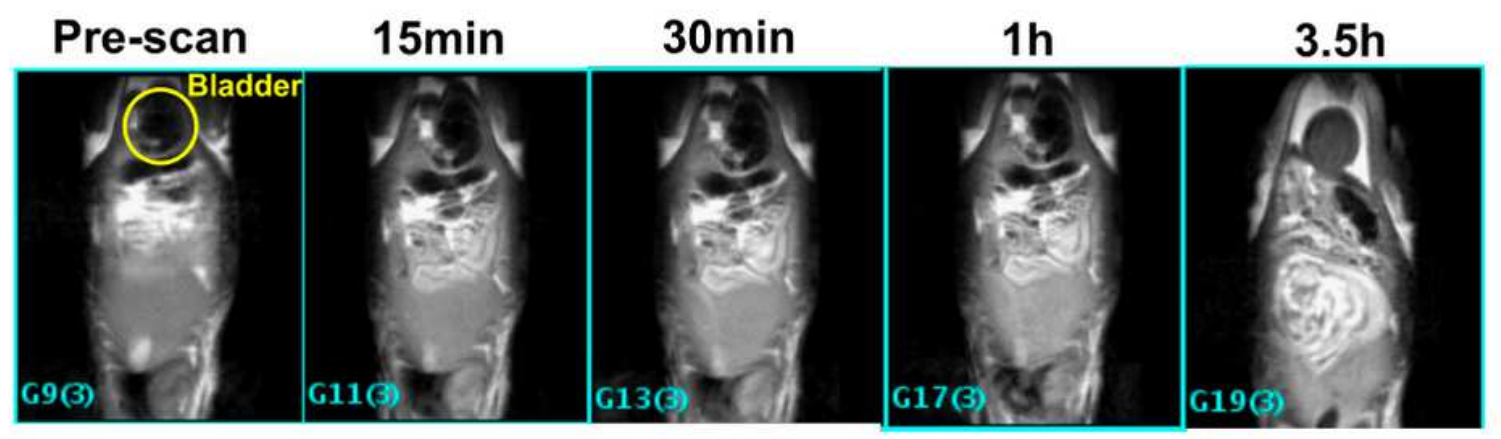

(b)

Temporal Evaluation (Gaussian)

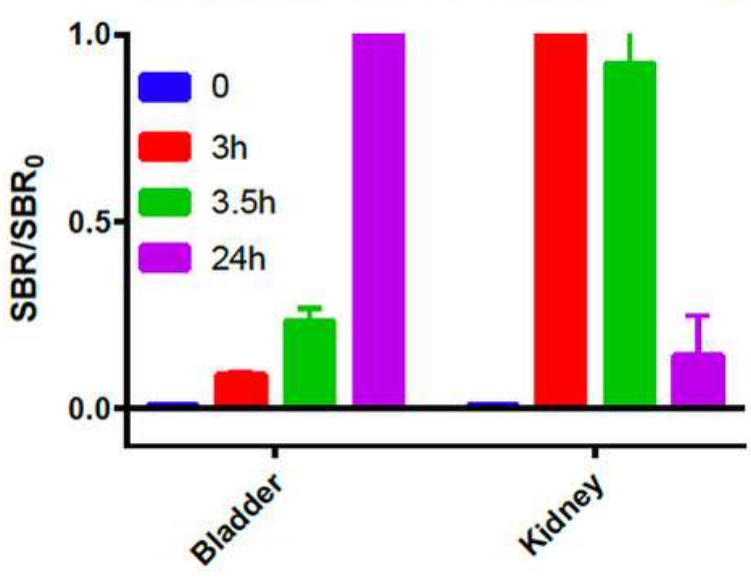

(c)

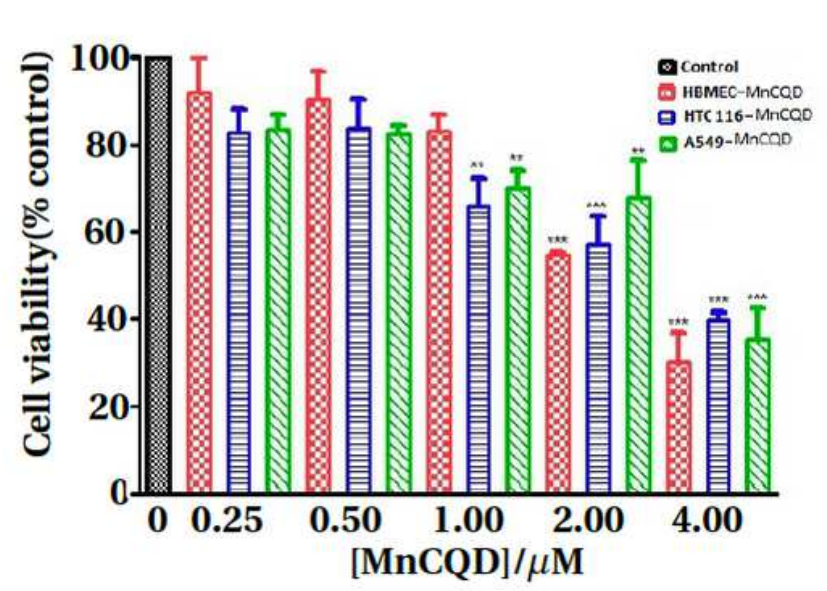

(d)

Figure 2

In vivo evaluation ofMnCQD as contrast agent showing the region of the kidneys (a) and the bladder (b). Biodistribution of the nanoparticles (c) in different mouse organ and cell viability assay (d) of MnCQD against the Human Brain Microvascular Endothelial cell (HBMEC), adenocarcinomic human alveolar basal epithelial cell (A549) and human colon cancer cell (HTC116) lines. 


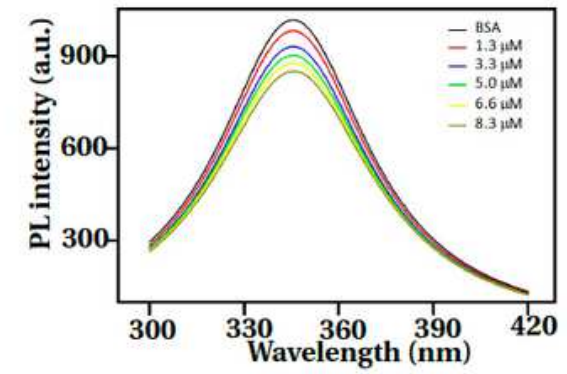

(a)

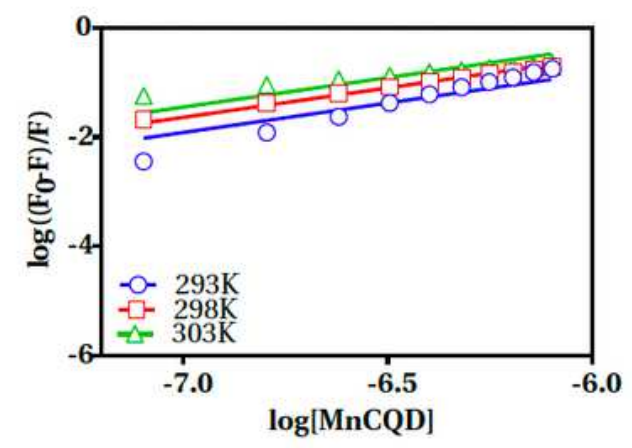

(c)

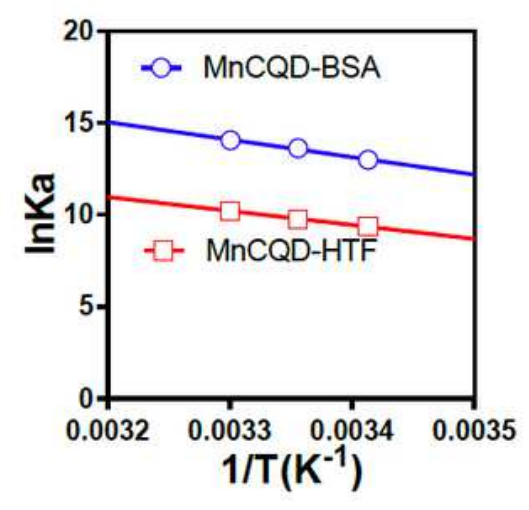

(e)

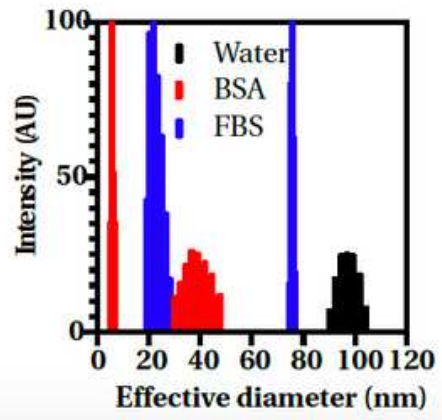

(g)

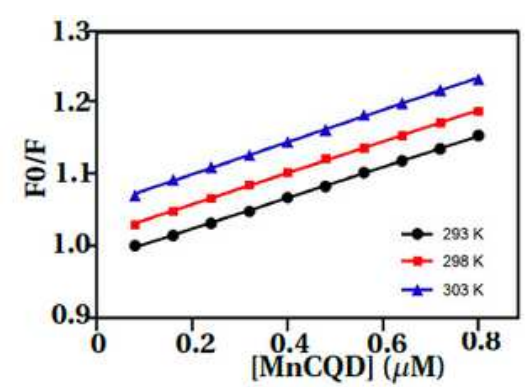

(b)

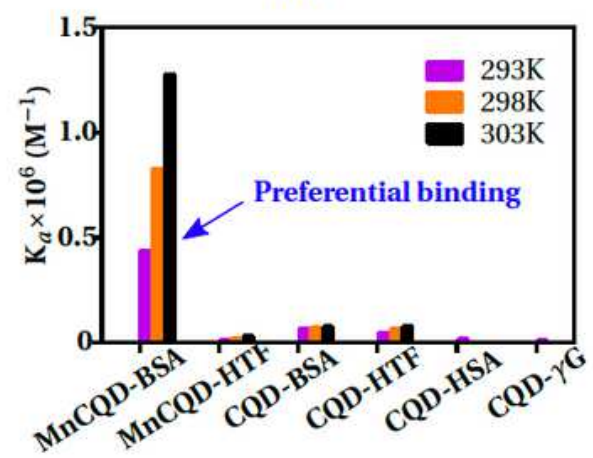

(d)

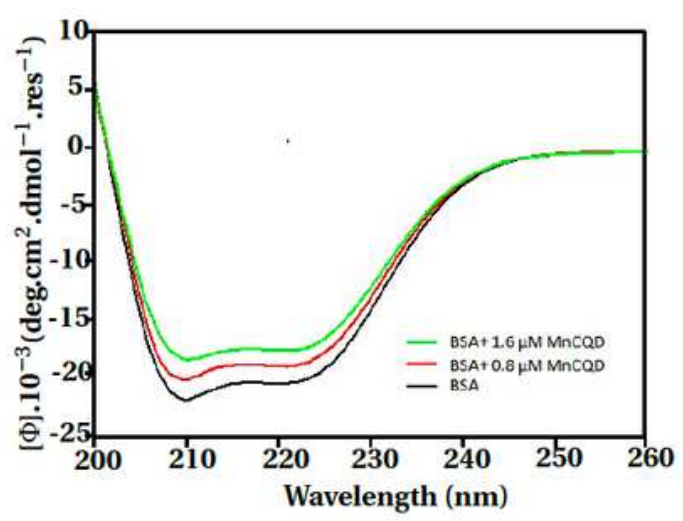

(f)

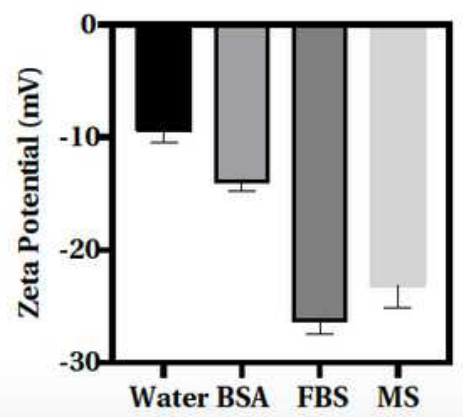

(h)

\section{Figure 3}

Fluorescence quenching experiments showing the fluorescence suppression of BSA (a) as theMnCQD are titrated to the proteins. Stern-Volmer plots (b), log plots (c) at three different temperatures, afinities comparison (d), Van't Hoff plots (e) used to determine the thermodynamic parameter. CD spectra of BSAMnCQD complexes (f ), DLS spectra (g) and zeta potential (h) of the MnCQD in water, BSA, FBS andMS. 


\section{Supplementary Files}

This is a list of supplementary files associated with this preprint. Click to download.

- GraphicalAbstract.png 